\title{
Evaluation of Viral Resistance to Reverse Transcriptase Inhibitors (RTI) in HIV-1- Infected Patients Before and After 6 Months of Single or Double Antiretroviral Therapy
}

Carlos Brites, Fabianna Bahia, Mark Gilbert, Célia Pedroso and Roberto Badaró

\author{
Retrovirus Laboratory, Professor Edgard \\ Santos Hospital, Federal University of Bahia, \\ Salvador, Bahia, Brazil
}

\begin{abstract}
We evaluated samples of peripheral blood mononuclear (PBMC) cells from 46 AIDS patients, before starting therapy with HIV-1 reverse transcriptase inhibitors (RTI), and after 6 months of drug use. PBMC were stored and tested by a Line Probe Assay (LiPA), in order to assess the frequency of RT mutations in this population. Six patients were taking AZT before initial blood collection ( 1 to 16 weeks of drug use) and 40 patients had no prior therapy. After baseline evaluation, 19 patients received AZT, 23 AZT plus DDI, 3 started AZT only with DDI added after 3 months, and 3 received a combination of AZT plus 3TC. Detection of at least one mutation was found in 33\% (15/46) of patients at baseline, and 83\% (38/46) had at least 1 mutation after 6 months of therapy. In the majority of cases, samples presented with the wild type and variants of HIV, simultaneously. Patients receiving monotherapy had a higher frequency of mutations (L41 and F214, Y215) than did patients receiving double-drug therapy (19 vs. 10). No specific mutation associated with DDI was identified in 26 patients so treated. Despite the finding of a mean increase in $\mathrm{CD}_{4}$ count and a mild decrease in viral load, patients tended to have an inverse correlation between the $\mathrm{CD}_{4}$ variation and number of mutations detected after 6 months, suggesting potential loss of drug efficacy in the presence of these genotypic changes.
\end{abstract}

Key Words: HIV-1, viral resistance, Brazil.

Development of viral resistance to RTI is a common finding among patients using sub optimal anti-retroviral (ARV) therapy [1-6]. In Brazil, only recently, triple therapy including the use of protease inhibitors (PI) became the standard of care for HIV-1 infected patients. The current guidelines established by the Ministry of Health still permit the use of double therapy in specific situations, such as intolerance to PI, or for those patients presenting with low viral load or high $\mathrm{CD}_{4}$ counts [7].

Received on 19 March 2001; revised 19 July 2001.

Address for correspondence: Dr. Carlos Brites. Rua João das Botas, s/n, $6^{\circ}$ andar, HUPES - Canela - Salvador, Bahia, Brazil. Phone (55 71) 235-4901.

This work was partially supported by a grant from Immunex Corp., Seattle, WA, USA, and by Brazilian Ministry of Health. Presented in part as a poster at The XIV International AIDS Conference, Durban, South Africa.

The Brazilian Journal of Infectious Diseases 2001;5(4):177-182 (C) 2001 by The Brazilian Journal of Infectious Diseases and Contexto Publishing. All rights reserved.

1413-8670
The high replication rate of HIV-1, associated with its high genetic variation, allows the onset of viral mutations that confers resistance to all anti-retroviral drugs currently available [8-11]. The selection of mutant viruses displaying such genotypic changes is a consequence of incomplete suppression of viral replication, and is also the major cause of anti-retroviral therapy failure [1].

We evaluated the genetic profile of HIV-1 RT in peripheral blood mononuclear cells (PBMC) samples of a group of 46 AIDS patients who had received no previous treatment with PIs and were drug naïve, or had prior use of only 1 RTI drug. Genetic analysis was attempted by using a line probe assay (LiPA) to determine the prevalence of RTI mutations at baseline and after 6 months of treatment with 1 or 2 drugs.

\section{Material and Methods}

Patients recruitment and study location

This collection of blood samples for this study was 
conducted between July, 1995, to June, 1997, at the Federal University of Bahia Hospital (HUPES). Patients with a previous diagnosis of AIDS had blood samples collected after a written informed consent was given. All patients had a diagnosis of AIDS established within 3 months of blood collection and were assigned to receive anti-retroviral drugs. Six patients already using 1 RTI for less than 16 weeks were also included in order to assess any difference in RT mutations related to a short term use of RTIs. All of the patients had HIV infection confirmed by Western Blot.

\section{Laboratory evaluation}

Viral load was measured at baseline and after 6 months by NASBA (Organon-Teknika) with a sensitivity of 400 RNA copies/ml of plasma. Determination of $\mathrm{CD}_{4}$ and $\mathrm{CD}_{8}$ cell counts was attempted by flow cytometry. Assessment of viral mutations was performed by using a Line Probe Assay (LiPA - Murex-Innogenetics) capable of detecting mutations at codons 41, 69, 70, 74, 184, 214 , and 215 , according to the manufacturer's instructions [12].

Briefly, PBMC were separated by Fycol Hipaque gradient of centrifugation, and stored in liquid nitrogen until processing. The cells were washed, adjusted to 2 million/ml, and centrifuged. The resulting pellet was lysed using the Amplicor-monitor lysis kit and a fragment of $1.2 \mathrm{~Kb}$ was amplified by PCR. A second PCR round using an inner primer was performed, and the final product was then hybridized with specific oligonucleotide probes, immobilized as parallel lines on membrane-based strips. After hybridization, streptavidine labeled with alkaline phosphatase was added and bound to any biotinylated hybrid previously formed. Incubation with BCIP/NBT chromogen results in a purple/brown precipitate.

\section{Statistical analysis}

A comparison of mean $\mathrm{CD}_{4}$ count and viral load for patients presenting or not presenting with RT mutations was carried out using analysis of variance. Percentages were compared Chi-square test.

\section{Results}

Patient Population and anti-retroviral regimen used

A total of 46 patients were enrolled in the study. Only 6 patients were using AZT or entry. Four patients were using AZT for 4 weeks, and the third and fourth patients used the drug for 4 and 8 weeks, respectively. The remaining 40 subjects were anti-retroviral drug naïve. Nineteen patients started therapy with AZT alone, 23 subjects received AZT plus DDI, and 3 initially received only AZT with DDI added to their prescription after 3 months. Only 1 patient was treated with a combination of AZT plus 3TC.

\section{Baseline evaluation}

At baseline, 13/40 (33\%) drug-naïve patients had at least $1 \mathrm{RT}$ mutation detected (Table 1). Six patients were already taking AZT. Two (2/6) of those patients had taken AZT for 8 weeks and had 2 detectable mutations (R70 and 184V for the first patient, R70 and F214/Y215 for the second). The other 4 patients under AZT therapy, but presenting with no detectable mutations were using that drug for only 1 week $(\mathrm{P}=0.003)$. Of note, $13 / 15$ patients with detected mutations also showed wild type pattern for the codons affected by genetic changes. The mean VL at baseline was $4.9 \pm 1.3 \log _{10}$ HIV-1 RNA copies/ml (median $5.0 \mathrm{log})$. Mean CD4 count was $126 \pm 80$ cells $/ \mathrm{ml}$ (median 114 cells/ml).

\section{Six months evaluation}

After 6 months of ARV drug use, $83 \%$ of patients had at least 1 RT mutation detected: $40 \%$ presented 1 RT mutation, $32 \%$ had 2 mutations, $9 \%$ developed 3 mutations and $2 \%$ presented with 4 mutations at LIPA. Again, we found a high frequency of samples presenting wild type and viral variants in the same test. Table 1 summarizes the genotypic profile of patients at baseline and after 6 months. Although we observed a random frequency of mutations for each therapy regimen utilized, mutations L41 (TTG) and F214/F215 were more prevalent among patients using AZT as a single drug than among those using 2 associated drugs (Table 2). 
Table 1. Frequency of RT codons detected by LiPA at baseline, and after 6 months, in 46 samples tested

\begin{tabular}{lccr}
\hline & $\begin{array}{c}\text { Baseline } \\
\text { N ( \%) }\end{array}$ & & $\begin{array}{c}\text { 6 months } \\
\mathbf{N}(\mathbf{\%})\end{array}$ \\
\hline M41 & $38(83)$ & WT* & $36(78)$ \\
L41(TTG) & - & Mut & $8(18)$ \\
L41(CTG) & $1(02)$ & Mut & $4(9)$ \\
T69, K70 & $42(91)$ & WT 3 & $9(85)$ \\
T69, R70 & $12(26)$ & Mut & $21(47)$ \\
K70, D69 & - & Mut & - \\
R69, R70 & - & Mut & - \\
N69, R70 & - & Mut & - \\
L74 & $33(73)$ & WT & $35(76)$ \\
V74 & - & Mut & - \\
M184 & $41(91)$ & WT & $39(85)$ \\
V184 & $1(2)$ & Mut & $3(7)$ \\
F214,T215 & $32(70)$ & WT & $31(69)$ \\
L214,T215 & $1(2)$ & WT & $1(2)$ \\
T215 & - & WT & $12(28)$ \\
F214,Y215 & $3(7)$ & Mut & $23(50)$ \\
L214,Y215 & - & Mut & - \\
F215,F215 & - & Mut & $1 \quad(2)$ \\
\hline
\end{tabular}

*WT=Wild type; Mut=Variant.

Table 2. Frequency of new mutations after 6 months of therapy, according to the RTI regimen used by patients

\begin{tabular}{lcccccc}
\hline & \multicolumn{6}{c}{ Variant codon } \\
\cline { 2 - 7 } Drugs & T69R70 & F214Y215 & L41(TTG) & V184M & F214F215 & L41(CTG) \\
\hline AZT & 4 & 11 & 6 & - & - & 2 \\
AZT+DDI & 3 & 6 & - & 1 & 1 & 1 \\
AZT+3TC & - & - & - & 1 & - & - \\
AZT $=>$ DDI & 2 & 3 & 2 & - & - & - \\
\hline
\end{tabular}


Figure 1. Mean variation of $\mathrm{CD}_{4}$ count after 6 months, according to the number of detected mutations (Mut)

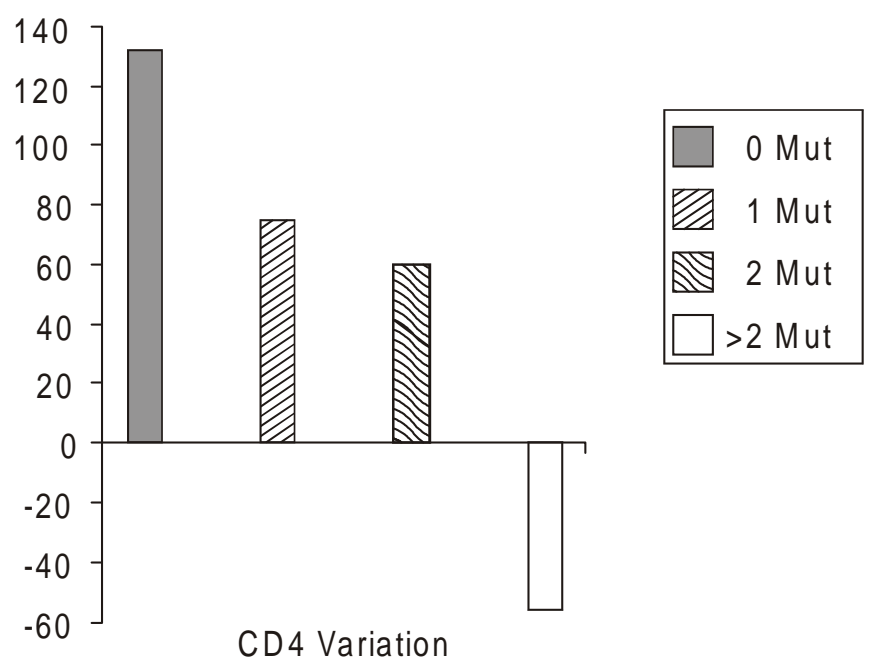

Mean viral load decreased to $4.3 \pm 1.8 \log _{10} \mathrm{HIV}-1$ RNA (median $4.8 \log _{10}$ ), while $\mathrm{CD}_{4}$ counts increased to $209 \pm 170$ cells/ml (median 186 cells/ml). However, patients who developed RT mutations during that period showed a trend for an inverse correlation between $\mathrm{CD}_{4}$ count and number of RT mutations after 6 months (Figure 1).

\section{Discussion}

Resistance to ART is considered an important cause of treatment failure for HIV-infected patients [13-15]. There is clear evidence that sub-optimal therapy resulting in insufficient viral suppression is followed by selection of mutants resistant to the ongoing therapy, leading to virological, immunological and clinical failure [1]. In consequence, patients using less than 3 ARV drugs (highly active anti-retroviral therapy - HAART) are likely to fail treatment over time [16-17].

Currently, treating HIV-1 infection with only 1 or 2 drugs is not considered a good strategy. However, use of AZT alone is still the standard of care for prophylaxis for mother-to-child transmission during pregnancy [1819]. Considering the need to keep pregnant women on an AZT single drug regimen, for prolonged periods, it raises a question about the risk of selecting mutant viruses due to incomplete viral suppression in the mother on prophylaxis. On the other hand, there is great concern about the risk of transmission of resistant viruses from patients heavily pre-treated and failing therapy, to other individuals. This fact has been reported in different countries and can become an important epidemiological problem in areas where resistant viruses are prevalent [20-24]. Thus, it is important to know about the prevalence of mutation in both patients failing therapy and drug-naïve individuals in order to evaluate the best initial treatment regimen, and to asses the potential efficacy of the current prophylaxis regimens for pregnant women.

This study detected a high prevalence of virus displaying genetic changes associated with resistance to AZT, even for patients not previously exposed to ARV drugs. In addition, the development of mutations in almost all individuals confirms the reported data about the inefficacy of therapeutic regimens using 1 or 2 RTI for treating patients with advanced disease. It is important to note the coexistence of wild type mutant patterns in a large proportion of such individuals. That 
finding could mean that the patients were starting to fail therapy, since part of the viral populations they harbored were sensitive to ARV drugs. However, the inverse correlation detected between viral load, $\mathrm{CD}_{4}$ count, and number of RT mutations indicates that there can be a potential clinical impact for the individuals under such therapy.

Another interesting point is the high rate of codon detection by LiPA in the present study. In particular, we were concerned about the sensitivity of this test for codon 41 , since a lower rate of detection was expected for this codon. In addition, it is important to note that no specific mutation associated to DDI use was detected, regardless of the fact that 26 subjects were using that drug during the follow up period. Thus, in this population, development of mutations associated to AZT were much more frequent than to DDI, although the changes in surrogate markers for HIV infection were no different for the patients using different regimens. Patients receiving double therapy showed similar rates of mutation development during the study period, but those under monotherapy had a trend for a higher frequency of some specific mutations (L41, and F214, Y215), as shown in Table 2.

We cannot rule out the possibility that the high rate of genetic changes detected by LiPA in this study do not reflect the true level of viral resistance to AZT, but only a high grade of HIV polymorphism in regions closely related to the codons detected by the assay, as suggested by studies comparing LiPA with viral sequencing. The variation in the markers of HIV infection reinforces the significance of these changes.

Our findings indicate that genetic evidence of AZT resistance is high among drug-naïve patients in Bahia. The use of 1 or 2 drugs for treating AIDS patients was associated with development of mutations associated with AZT resistance in almost all patients tested. The simultaneous detection of wild type and variant viruses may indicate that patients could still partially respond to the drugs in use, but the variation of the markers of disease progression (VL/CD 4 count) after 6 months do not support this hypothesis. Our findings suggest that primary viral resistance may be high among drug- naïve patients in Bahia, at least among patients with advanced stage of disease. The potential impact of these findings on HAART efficacy, as well as for prophylaxis in pregnant women, deserves further investigations.

\section{References}

1. Moyle G. J. Resistance to antiretroviral compounds: implications for the clinical management of HIV infection. Immunology \& Infectious Diseases 1995;5:170-82.

2. Larder B. A., Kemp S. D. Multiple mutations in HIV-1 reverse transcriptase confer high-level resistance to zidovudine (AZT). Science 1989;246:1155-8.

3. Shirasaka T., Kavlick M., Ueno T., et al. Emergence of human immunodeficiency virus type 1 variants with resistance to multiple dideoxynucleosides in patients receiving therapy with dideoxynucleosides. Proceedings of the National Academy of Sciences USA 1995;92:2398-402.

4. Schmit J., Cogniaux J., Hermans P., et al. Multiple drug resistance to nucleoside analogues and nonnucleoside reverse transcriptase inhibitors in an efficiently replicating human immunodeficiency virus type 1 patient strain. J Infect Dis 1996; 174:962-8.

5. Shafer R., Kozal M., Winters M., et al. Combination therapy with zidovudine and didanosine selects for drugresistant HIV-1 strains with unique patters of pol gene mutations. J Infect Dis 1994;169:722-9.

6. Shafer R., Iversen A., Winters M., et al. Drug resistance and heterogeneous long-term virologic responses of HIV type 1-infected subjects to zidovudine and didanosine combination therapy. J Infect Dis 1995; 172:70-8.

7. Ministério da Saúde do Brasil. Recomendações para terapia antiretroviral (ARV) em adultos e adolescentes infectados pelo HIV, 2000.

8. Iversen A.K.N., Shafer R.W., Wehrly K., et al. Merigan. Multidrug-Resistant Human Immunodeficiency Virus Type 1 Strains Resulting from Combination Antiretroviral Therapy. J Virol 1996;70(2):1086-90.

9. Hirsch M.S., Conway B., D'Aquila R.T., et al. Antiretroviral Drug Resistance Testing in Adults With HIV Infection. JAMA 1998;276(24):1984-91.

10. Yerly S., Kaiser L., Race E., et al. Transmission of antiretroviral-drug-resistant HIV-1 variants. Lancet 1999;345:729-33.

11. Boden D., Hurley A., Zhang L., et al. HIV-1 Drug Resistance in Newly Infected Individuals. JAMA 1999;282(12):1135-41. 
12. Stuyver L., Wyseur A., Rombout A., et al. Line Probe assay (LiPA) for the detection of drug-selected variants in the HIV-1 reverse transcriptase gene. International Antiviral News 1997;5(3):38-40.

13. Richmann DD. Resistance of clinical isolates of human immunodeficiency virus to ARV agents. Antimicrob Agents Chemother 1993;37:1207-13.

14. Erice A., Belfour H. Resistance of human immunodeficiency virus type 1 to ARV: a review. Clin Infect Dis 1994; $18: 149-56$.

15. Kozel M.J., Shafer R.W., Winter M.A., et al. A mutation in human immunodifeciency virus $\mathrm{RT}$ and decline in $\mathrm{CD}_{4}$ lymphocyte numbers in longterm zidovudine recipients. J Infect Dis 1993; 167:526-32.

16. Palella F.J., Delaney K.M., Moorman A.C., et al. Declining morbidity and mortality among patients with advanced human immunodeficiency virus infection. N Engl J Med 1998; 338: 853-60.

17. Carpenter C.C.J., Cooper D.A., Fischl M.A., et al. Antiretroviral therapy for HIV infections. Updated recommendations of the International AIDS Society USA panel. JAMA 2000;283:381-90.

18. Public Health Service Task Force recommendations for the use of antiretroviral drugs in pregnant women infected with HIV-1 for maternal health and for reducing perinatal HIV-1 transmission in the United States. MMWR 1998;47(RR-2):1-30.
19. Wade N.A., Birkhead G.S., Warren B.L., et al. Abbreviated regimens of zidovudine prophylaxis and perinatal transmission of the human immunodeficiency virus. $N$ Engl J Med 1998;339(20):1409-14

20. Parkin N.T., Lie Y.S., Hellmann N., et al. Phenotypic changes in drug susceptibility associated with failure of human immunodeficiency virus type 1 (HIV-1) triple combination therapy. J Infect Dis 1999; $180: 865-70$.

21. Villalba N., Gómez-Cano M., Holguín A., et al. Multiple drug resistance genotype causing failure of antiretroviral treatment in an HIV-1 infected patient heavily exposed to nucleoside analogues. Eur J Clin Microbiol Infect Dis 1999; 18:372-5.

22. Lepri A.C., Sabin C.A., Staszewski S., et al. Resistance profiles in patients with viral rebound on potent antiretroviral therapy. J Infect Dis 2000; 181:1143-7.

23. Little S.J., Daar E.S., D'Aquila R.T., et al. Reduced antiretroviral drug susceptibility among patients with primary HIV infection. JAMA 1999;282(12):1142-9.

24. Tamalet C., Pasquier C., Yahi N., et al. Prevalence of drug resistant mutants and virological response to combination therapy in patients with primary HIV-1 infection. J Med Virol 2000;61:181-6. 